\title{
Report on Space-Qualified Readout Electronics for the BGO Calorimeter of DAMPE Mission
}

\section{Changqing FENG ${ }^{1}$}

Department of Modern Physics, University of Science and Technology of China State Key Laboratory of Particel Detection and Electronics (IHEP-USTC)

Hefei, 230026, China

E-mail: fengcq@ustc.edu.cn

\section{Siyuan MA}

Department of Modern Physics, University of Science and Technology of China State Key Laboratory of Particel Detection and Electronics (IHEP-USTC)

Hefei, 230026, China

E-mail: siyuanma@mail.ustc.edu.cn

\section{Di YANG}

Department of Modern Physics, University of Science and Technology of China State Key Laboratory of Particel Detection and Electronics (IHEP-USTC)

Hefei, 230026, China

E-mail: dyg87email.ustc.edu.cn

\section{Qi WANG}

Department of Modern Physics, University of Science and Technology of China State Key Laboratory of Particel Detection and Electronics (IHEP-USTC)

Hefei, 230026, China

E-mail: wangmo@mail.ustc.edu.cn

\section{Deliang ZHANG}

Department of Modern Physics, University of Science and Technology of China State Key Laboratory of Particel Detection and Electronics (IHEP-USTC)

Hefei, 230026, China

E-mail: dlzhang@mail.ustc.edu.cn

\section{Shubin LIU*}

Department of Modern Physics, University of Science and Technology of China State Key Laboratory of Particel Detection and Electronics (IHEP-USTC)

Hefei, 230026, China

E-mail:liushb@ustc.edu.cn

\section{Qi AN}

Department of Modern Physics, University of Science and Technology of China State Key Laboratory of Particel Detection and Electronics (IHEP-USTC)

Hefei, 230026, China

\footnotetext{
${ }^{1}$ Speaker; ${ }^{*}$ Corresponding Author

(c) Copyright owned by the author(s) under the terms of the Creative Commons Attribution-NonCommercial-ShareAlike Licence.
} 


\begin{abstract}
A satellite-borne high energy cosmic ray detector planned to be launched in the end of 2015, named DArk Matter Particle Explorer (DAMPE), is being developed in China. The BGO Calorimeter, which contains 308 BGO (Bismuth Germanate) crystal bars and 616 PMTs (photomultiplier tubes), is a critical sub-detector for measuring the energy of cosmic particles, distinguishing positrons/electrons and gamma rays from hadron background, and providing trigger information. It contains 1848 signal channels and a complex readout system with 16 front-end electronic (FEE) boards. From year 2013 to 2015, a qualification model and a flight model for the BGO Calorimeter, together with its readout electronics, were successfully developed and passed ground-based cosmic ray tests and a series of qualification level environmental tests. Four test beam experiments for the DAMPE Qualification Model were carried out with the CERN PS and SPS facilities, which proved the major specifications of the BGO Calorimeter, such as energy range and resolution, and the capability of its readout electronics. The progress and some details related to the high reliability design and quality control for the readout electronics will be introduced in this paper.
\end{abstract}

Keywords: Dark Matter, DAMPE, Calorimeter, BGO

The 34th International Cosmic Ray Conference

30 July- 6 August, 2015

The Hague, The Netherlands 


\section{Introduction}

In recent years, to observe the high energy primary cosmic rays in space is a hot topic in the physics community, which is regarded to be related to the clue of dark matter. Several experiments have already been successfully conducted, such as the ATIC Antarctic Baloon [1], the Fermi/LAT satellite [2], the PAMELA satellite [3] and the AMS02 [4].

In China, a scientific satellite named DAMPE (DArk Matter Particle Explorer) is being developed, with a $500 \mathrm{~km}$ orbit altitude and a designed mission period of more than 3 years. The project was proposed mainly by Purple Mountain Observatory of CAS (Chinese Academy of Sciences), while University of Science and Technology of China is one of the major collaborators. The main scientific objectives of DAMPE are cosmic ray study, gamma ray astronomy, and searching for the clue of dark matter particles by investigating the composition and energy spectra of primary cosmic rays, especially positrons, electrons and gamma rays over an energy range from $5 \mathrm{GeV}$ to $10 \mathrm{TeV}$.

As a critical sub-detector with the functions of measuring the energy of cosmic ray particles, distinguishing positrons/electrons and gamma rays from hadron background, and providing trigger information, the BGO Calorimeter is composed of 308 BGO (Bismuth Germanate Oxid) crystal bars, with the size of $2.5 \mathrm{~cm} \times 2.5 \mathrm{~cm} \times 60 \mathrm{~cm}$ for each bar. Each crystal bar is viewed by two Hamamatsu R5610A-01 PMTs (photomultiplier tubes) from both sides. According to physics simulation, a dynamic range of about $2 \times 10^{5}$ is required for each BGO bar, which obviously exceeds the capability of one single front-end electronics channel. Therefore a three-dynode (2, 5 and 8) scheme for the PMT base design is proposed [5], resulting in 1848 readout channels for the 616 PMTs in total.

Fig. 1 illustrates the system configuration for the BGO detector and its readout electronics. Three types of FEE (Front-End Electronics) modules are designed, named FEE-A, FEE-B and FEE-C. FEE-A and FEE-B, both receive the 132 input channels in two layers; while FEE-C, with 66 input channels, only reads out one single layer. Besides precisely measuring the charge of PMT signals, the FEE-A module (for reading out Layer 1, 2, 3, 4, 11, 12, 13 and 14) needs to provide hit signal for the PDPU (Payload Data Process Unit) crate. In total, there are 16 FEE modules used for the BGO detector, with 4 modules assembled on each side.
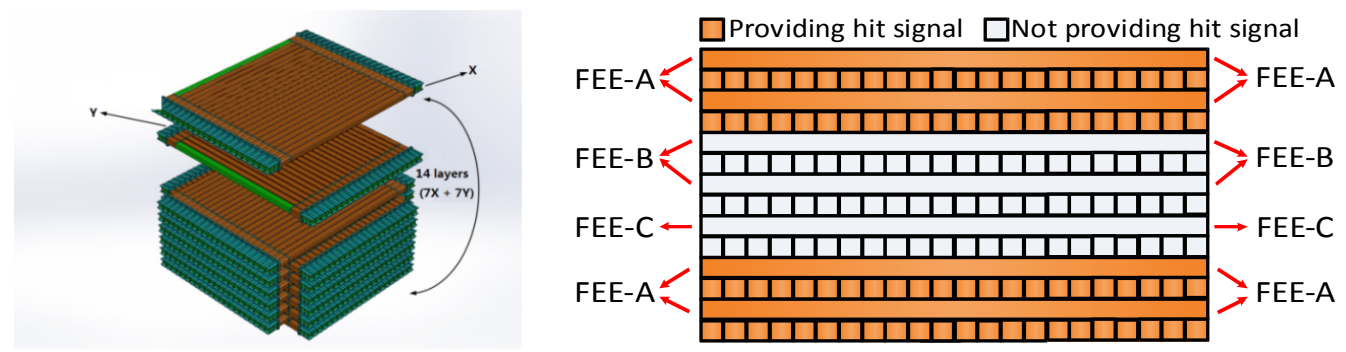

Fig. 1. Arrangement for BGO crystal bars (left) and configuration for the BGO detector and FEEs (right)

The photograph of one BGO FEE module (Type A, qualification-model) is shown in Fig. 2. Three ASICs (named VA160 and VATA160) are mounted on top side while the other three ones are mounted on bottom side. PMT signals from dynode 2, 5 and 8 are sent to the three ASIC (top or bottom side) from left to right respectively. The control logic of FEE module is implemented in an Actel flash-based ProASIC Plus FPGA (APA600 for FEE-A and APA300 
for FEE-B/C).

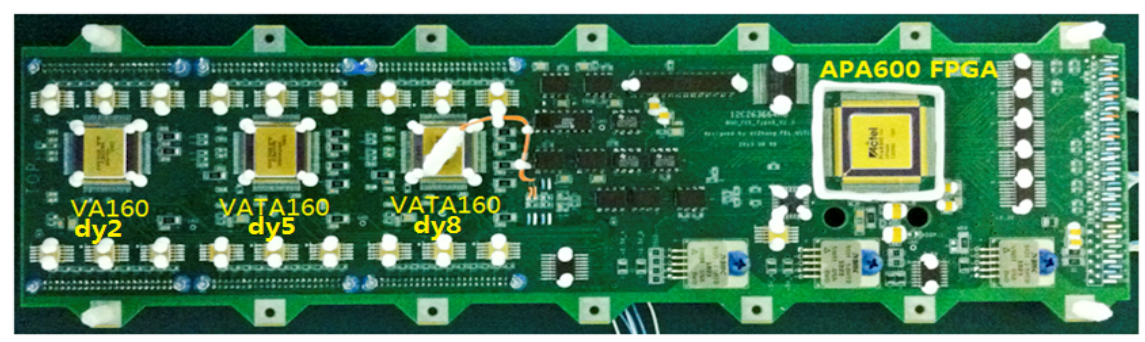

Fig. 2. Photograph of the qualification-model of an FEE module (Type A)

As a space instrument with a 3-year mission period, stringent requirements are imposed on the design and production process of the readout electronics for BGO calorimeter. Some of the critical issues, such as quality assurance for ASIC components, radiation hardening design, thermal design and board level screening tests.

\section{Quality assurance for ASIC components}

Two types of front-end ASICs, named VA160 and VATA160, are adopted for the frontend electronics of $\mathrm{BGO}$ calorimeter to perform the charge measurement function and to provide "hit" information of the crystal bars. There are 2 VA160 and 4 VATA160 chips used in an FEEA module, 6 VA160 chips used in an FEE-B module and 3 VA160 chips used in an FEE-C module, while totally 52 VA160 and 32 VATA160 chips are used for the BGO calorimeter.

The VA160 and VATA160 ASICs, optimized for the PMT dynode signals of DAMPE payload (for Plastic Scintillation Detector and BGO Calorimeter), were designed by IDE AS Inc. in Norway [6] and fabricated with AMS 0.35um CMOS process, in 2013. The wafers were thinned and cut into dies and later the bare dies were shipped from Norway to China, with antistatic vacuum packaging.

In order to comply with the quality control regulations for aerospace electronics, firstly 8 samples from each die lot were submitted for SEM (scanning electron microscope) check, with the acceptable rejection rate of $0 \%$. Secondly visual inspection for bond pads and glassivation layer was conducted for all the dies. And only the dies that passed the visual inspection were bonded in a ceramic package, which is shown in Fig. 3.
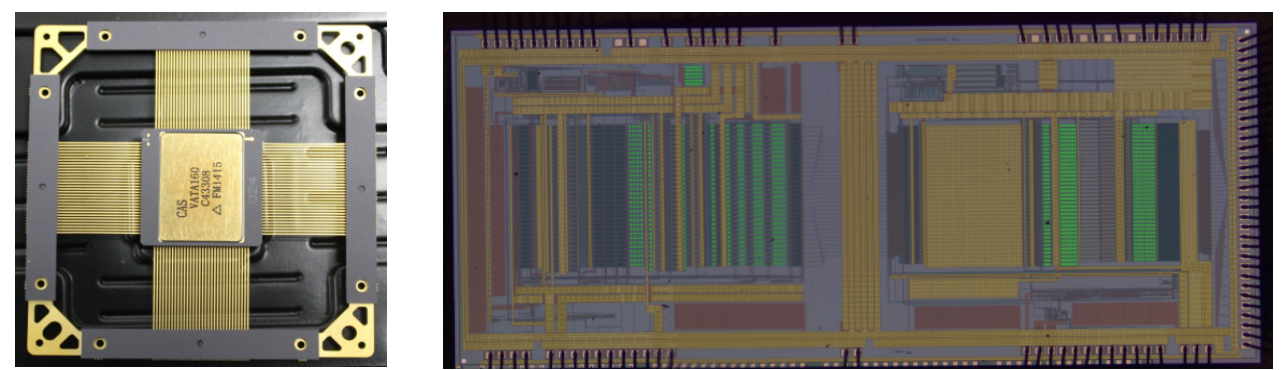

Fig. 3. Left: The ceramic package for the VA160 and VATA160 ASICs; Right: Microscope photograph of an VATA160 ASIC die after being bounded in a ceramic package

Finally it turned out that the rejection rate of visual inspection is about $20 \%$ for all the 430 VA160 and 230 VATA160 dies ordered for DAMPE project, which is better than the acceptance level sated in the contract (less than 30\%). In fact, most of the rejections reasoned from some minor flaws, such as tiny mechanical damage, or fracture most probably caused by wafer cutting. The imperfects are usually near the protecting boundary and can even be accepted 
by other ordinary applications, as shown in Fig. 4 (left). Others were rejected because of disfigurements maybe caused by manufacturing process, as illustrated in Fig. 4 (right).
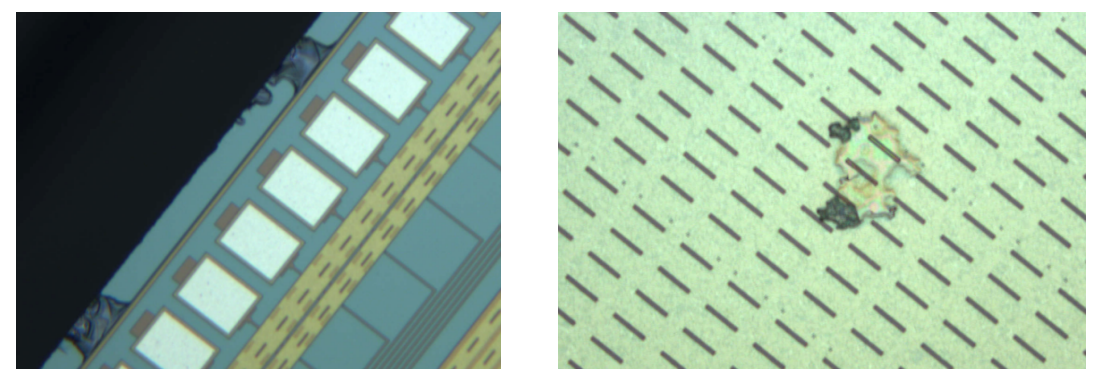

Fig. 4. Microscope photograph of the imperfect ASIC dies

After bonding, a series of lot screening for VA160 and VATA160 were carried out, according to the appendix A of GJB-548B-2005. Fig. 5 shows the screening procedures for VA160 and VATA160. In addition, in the Flight Model Stage, 22 VA160 and 22 VATA160 ASIC chips which passed the screening were submitted for 1000-hour test at the temperature of $125^{\circ} \mathrm{C}$, with the acceptable rejection rate of $0 \%$.

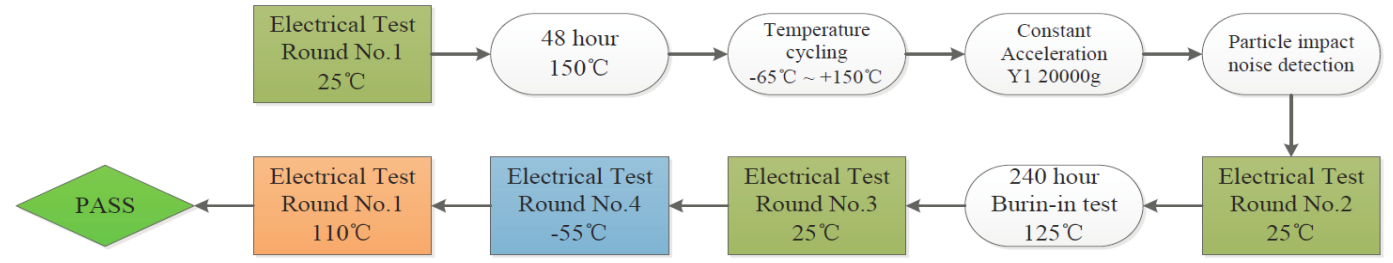

Fig. 5. Screening procedures for VA160 and VATA160

Considering that there were several hundred ASIC chips needing screening, an automatic test platform was designed [7], which can quickly obtain the key performances of the chips and record the results. With the help of this test platform, we successfully completed the screening procedures of nearly 400 VA160 chips and 200 VATA160 chips in total, for DAMPE project.

It turned out that very few chips failed the screening, and all the chips which passed the screening behave stable after being assembled in BGO FEEs.

\section{Radiation-Harden Design}

The DAMPE satellite is planned to operate continuously in an orbit with the attitude of $500 \mathrm{~km}$ and an inclination of 97 degrees. The expected mission period is more than 3 years. However, the radiation environment in space is a critical threat to its reliability.

According to simulation, with $3 \mathrm{~mm}$ aluminum shielding, the total dose during 3 years in the DAMPE orbit is less than $3 \mathrm{krad}$, which is much lower than the tolerance level of the components applied for BGO readout electronics. Furthermore, the real condition is much more optimistic benefiting from the large mass (approximately $1000 \mathrm{~kg}$ ) of BGO crystals and structures, which can provide ideal shielding for the FEE components.

But the SEE (Single Event Effect), including SEL (Single Event Latch-up) and SEU (Single Event Upset), is a main radiation effect that the semiconductor components of BGO electronics may suffer. The DAMPE orbit has a wide variety of particles with various kinetic energies, from galactic cosmic rays, solar particles, and particles trapped in Van Allen belts. A simulation shows that the integral flux declines more than three orders of magnitude rapidly when LET (Linear Energy Transfer) is around $30.0 \mathrm{MeV}-\mathrm{cm}^{2} / \mathrm{mg}$; if SEE threshold (in LET) of 
an ASIC component is higher than $37.5 \mathrm{MeV}-\mathrm{cm}^{2} / \mathrm{mg}$, the calculated SEE rate will be lower than $1.0 \times 10^{-8} /$ device/day.

In order to evaluate the reliability in the space radiation environment, a series of single event effect (SEE) tests were conducted, both for the ASICs and for the FPGA chips [8, 9]. Test results showed that both VA160 and VATA160 ASICs are sensitive to single event latch-up (SEL), thus effective SEL protection circuit was designed for the front-end electronics board. But SEU is not a serious problem, with TMR (triple module redundancy) design for the configuration registers in the ASIC design and proper sequential design for the readout operation.

\section{Thermal Design}

The importance of thermal design for the BGO electronics comes from two facts:

The first fact is that the BGO detector is sensitive to operating temperature [10], because the photon yield of BGO crystal and the gain of PMT are both proven to have negative temperature coefficients, about 1-2\% per centigrade in total. As the FEEs and detector units (PMTs and BGO bars) are assembled together closely, the operating temperature of the detector can be directly influenced by the heat produced by the FEEs.

The second fact is the simplified thermal design without active temperature control, mainly due to weight and power reduction strategy of the satellite platform.

A direct solution is cutting down the power dissipation of FEEs and increasing their thermal conductivity to the outer shielding panel. The latter is achieved by increase the contact area, and inserting a thin thermal conductive pad (Sil-Pad 2000 from the Bergquist Company, thickness: $0.254 \mathrm{~mm}$ ) between the FEE PCB board and the aluminum supporting structure. This solution can significantly reduce the temperature of both FEEs and the BGO detector units, which is regarded to be an effective way to ensure the lifetime of PMTs and to decrease their dark noise, as well as to increase the reliability of the components of FEE itself. According to test results, the total power consumption for the 16 BGO FEEs is $26 \mathrm{~W}$, which is less than the budget (less than $42 \mathrm{~W}$ ).

A simulation result using finite element analysis tools is given in Fig. 6. The boundary temperature is $20^{\circ} \mathrm{C}$ on the shielding panel. The results suggest that the thermal design concept of increase the contact area and inserting a thin thermal conductive pad is quite effective.
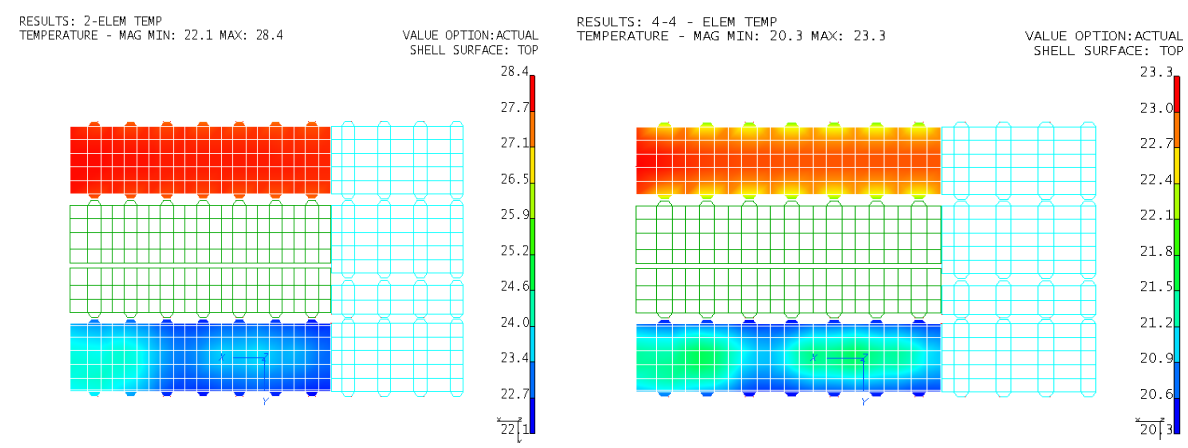

Fig.6. A thermal simulation result for BGO FEE PCB board

(Upper left: small contact area without thermal pad; Upper right: small contact area with thermal pad;

Lower left: Large contact area without thermal pad; Upper right: Large contact area with thermal pad; this figure is provide by Mr Liguo Wang, from Nanjing University of Aeronautics and Astronautics) 
For each FEE, a thermal resistor is attached to the lid of one VA160 or VATA160 package, while three other thermal resistors are attached to the PMTs or crystals. These thermal resistors are used to monitor the temperature status of electronics and detector units. The results from satellite-level thermal balancing tests showed that the temperature difference between the FEE chips and shielding panel is less than $10^{\circ} \mathrm{C}$, and the difference between the detector and shielding panel is less than $5^{\circ} \mathrm{C}$, which are consistent with the simulation results.

\section{Board Level Environmental Stress Screening}

The qualification model of the BGO calorimeter was assembled at the end of 2013, and the flight model was assembled at the beginning of 2015. For each model, 20 BGO FEE modules, including four spare ones, were soldered in a factory.

According to the quality control regulation of DAMPE project, each FEE module should pass a board-level ESS (environmental stress screening) procedure before assembly with the detector. The ESS procedure is composed of 16.5 thermal cycles, with the temperature level from $-45^{\circ} \mathrm{C}$ to $75^{\circ} \mathrm{C}$. The required rate of temperature change is great than $5^{\circ} \mathrm{C} / \mathrm{min}$ while the residence time at extreme temperature is about 2 hours.

The FEEs were placed in a oven with atmospheric pressure, and the performances of FEEs are tested at the temperature point of $-25^{\circ} \mathrm{C}$ and $+55^{\circ} \mathrm{C}$ (for the qulification model) or $+45^{\circ} \mathrm{C}$ (for the flight model) during cycling. Only the FEE modules which function normally and behave stable can be seen as passing the tests and can be submitted for assembly. Totally there are 40 BGO FEE modules for the DAMPE project (including the qualification model and the flight model) were mass produced, and all of them passed the ESS tests successfully, due to the strict quality control of components and board level assembly.
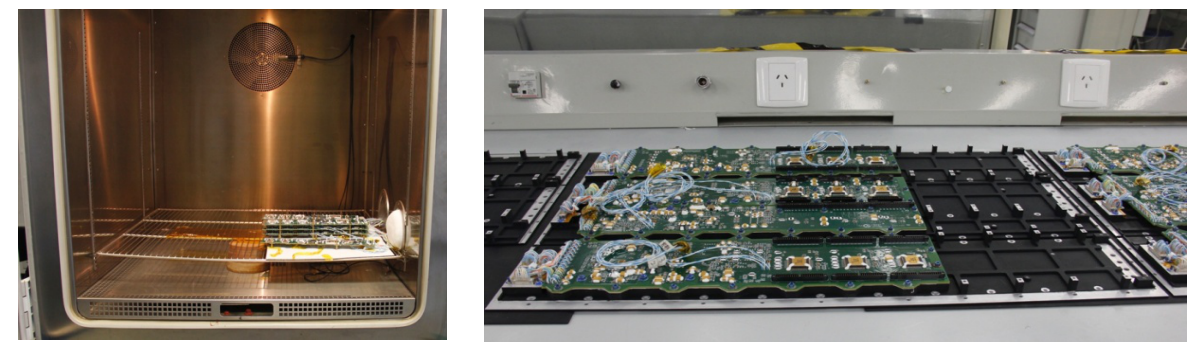

Fig. 7. Left: FEEs in the oven waiting for ESS tests; Right: FEEs assembled with shielding panel.

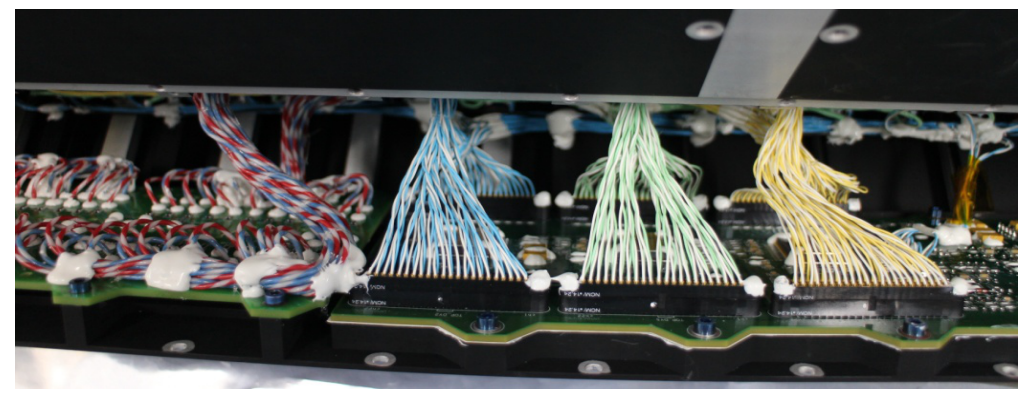

Fig. 8. The BGO FEE assembled with the detector (before closing the shielding panel)

\section{Conclusion}

From year 2013 to 2015, a qualification model and a flight model for the BGO 
Calorimeter, together with its readout electronics, were successfully designed and developed. The models passed the ground-based tests, including a series of environmental tests and integral tests with other sub-systems, and a series of test beam experiments (with the DAMPE Qualification Model).

During all the tests, the BGO readout electronics operate stable with the detectors, which indicate that the capability and reliability of the BGO electronics is realized.

Right now the environmental tests for DAMPE flight model are ongoing. The satellite is planned to be launched at the end of 2015 .

\section{Acknowledgement}

The authors would like to thank to Dr. Yunlong Zhang, Dr. Jianhua Guo, Mr Yiming Hu, Mr Dengyi Chen, Mr Zhe Li and Mr Weisong Ye for their helpful support and suggestions. We also thank Mr Liguo Wang for providing the figures of thermal simulation.

This work was supported by the Strategic Priority Research Program on Space Science of Chinese Academy of Sciences (Grant No. XDA04040202-4), and the National Basic Research Program (973 Program) of China (Grant No. 2010CB833002).

\section{References}

[1] T. G. Guzik, et al., The Advanced Thin Ionization Calorimeter (ATIC) for Studies of High Energy Cosmic Rays, the 26th International Cosmic Ray Conference, Salt Lake City, USA, August, 1999.

[2] W. B. ATWOOD, et al., The Large Area Telescope on the Fermi Gamma-ray Space Telescope Mission, Astrophys. J. 697:1071-1102, Feb 2009.

[3] P. Picozzaet al., PAMELA - A Payload for Antimatter Matter Exploration and Light-nuclei Astrophysics, arXiv:astro-ph/0608697v2, Jan 2007, Available: http://arxiv.org/pdf/astroph/0608697.pdf

[4] AMS Collaboration, M. Aguilar, et al., First Result from the Alpha Magnetic Spectrometer on the International Space Station: Precision Measurement of the Positron Fraction in Primary Cosmic Rays of 0.5-350 GeV, Phys. Rev. Lett., vol. 110, no.14, 141102, Apr 2013.

[5] Zhiyong Zhang, et al., Design of a high dynamic range photomultiplier base board for the $B G O E C A L$ of DAMPE, Nuclear Instruments and Methods in Physics Research Section A, Vol. 780, pp. 21-26, April 2015.

[6] VA160 datasheet and VATA160 datasheet, Integrated Detector Electronics AS (IDEAS), Nydalen, Oslo, Norway, 2013. http://www.ideas.no.

[7] Di Jiang, et al., Design of a Test Platform for Screening Procedures of VA160 and VATA160 ASICs in DAMPE Mission, 19th IEEE Real-Time Conference.

[8] GAO S. S., FENG C. Q., et al., Radiation tolerance studies on the VA32 ASIC for DAMPE BGO calorimeter, Nucl. Sci. Tech., 2014, 25(1):010402.

[9] GAO Shan-Shan, FENG Chang-Qing, et al., Single Event Effect Hardness for the Front-end ASICs Applied in BGO Calorimeter of DAMPE Satellite, Chinese Physics C, 2015, submitted.

[10] WANG PeiLong, ZHANG Yunlong, et al., Study on the temperature dependence of BGO light yield, Science China Physics, Mechanics \&Astronomy, vol. 57, no. 10, pp. 1898-1901, Oct. 2014. 